Research Article

\title{
Based on Artificial Intelligence in the Judicial Field Operation Status and Countermeasure Analysis
}

\author{
Kongze Zhu (iD) ${ }^{1}$ and Lei Zheng ${ }^{2}$ \\ ${ }^{1}$ Advanced Institute of Humanities and Social Sciences, Beihang University, Beijing 100191, Haidian, China \\ ${ }^{2}$ School of Electronic and Information Engineering, Beihang University, Beijing 100191, Haidian, China \\ Correspondence should be addressed to Kongze Zhu; zkz17605391002@buaa.edu.cn
}

Received 21 May 2021; Revised 22 July 2021; Accepted 4 August 2021; Published 14 September 2021

Academic Editor: Sang-Bing Tsai

Copyright (c) 2021 Kongze Zhu and Lei Zheng. This is an open access article distributed under the Creative Commons Attribution License, which permits unrestricted use, distribution, and reproduction in any medium, provided the original work is properly cited.

\begin{abstract}
Artificial intelligence is a recently emerging system that uses computers and big data as the basis to simulate human-like behavior with machines. Artificial intelligence is a way to imitate human thinking by learning massive data knowledge and using algorithms to reason and analyze the data. In the current age of advanced technology, many jobs in the justice system can be replaced by artificial technology technologies. Many courts have now scrutinized the use of artificial intelligence in the judiciary. With artificial intelligence, timely warnings on all aspects of admissions can effectively protect random or outdated trials and allocate social resources appropriately. In addition, it may better redress cases of misconduct and irregular conduct in the judiciary, which is conducive to justice. Based on BP neural network, research on related content and other methods has drawn relevant arguments, which will provide a certain theoretical basis for artificial intelligence to assist the judicial field in the future. The research in this article shows that artificial intelligence is conducive to suppressing duty crimes in the judicial field, promoting the transformation of extensive processing to intensive processing, and is conducive to judicial efficiency. In 2017, there were more than 8 million first-instance civil cases, but only 100,000 cases were closed. But by 2020, with the construction of smart courts, millions of cases out of more than 10 million first-instance civil cases are expected to be closed. The situation has been greatly improved. But at the same time, we also need to prevent the leakage of artificial intelligence to personal privacy, establish and improve corresponding laws and regulations, and coordinate the judgment relationship between the human brain and the machine brain. Artificial intelligence may be more suitable for assisting judicial judgments.
\end{abstract}

\section{Introduction}

With the release of the "New Generation Artificial Intelligence Development Plan" in our country, artificial intelligence has become more and more important, and it is more and more closely related to life [1]. Many large Internet companies and countries have begun to engage in artificial intelligence research, hoping to obtain a good foundation in the future, and the continuous development of artificial intelligence will continue to drive changes in all walks of life. Judicial administrative affairs unit is one of the important factors that constitute our nation and political power, and it also has an important position in our judicial system and the construction of the rule of law. The main functions of judicial administration include strengthening prison management, legal propaganda, mediation, community rehabilitation, residential assistance, legal services, certification of practicing lawyers, court appraisal, and judicial examinations. The creation of a judicial system and the modernization of judicial capabilities are also inevitable choices for the people's courts to adapt to the new wave of network technology innovation [2].

Foreign research on the development of artificial intelligence technology has also made some explorations. Rongpeng et al. conducted theoretical demonstrations on the application of artificial intelligence to service industries and industries that require related professional skills and studied how artificial intelligence organizes knowledge from 
the direction of big data [3]. Goyache et al. released the report "Artificial Intelligence: The Next Digital Frontier?" This article analyzes the attitudes and perceptions of various industries on artificial intelligence and gives a detailed report on how artificial intelligence is applied in fields including electromechanics, large supermarket chains, manufacturing, and education [4]. Krittanawong et al. believe that artificial intelligence technology will improve the efficiency of judicial work and change the effects of traditional work. The application of artificial intelligence technology can effectively analyze historical criminal records, summarize laws, and predict future crimes. They put forward the theory of "artificial intelligence driving justice" [5]. The forerunners have conducted detailed studies on the process of artificial intelligence development and the potential for application, but there are not many specific entries directly related to the use of the field of justice and potential issues.

From a philosophical point of view, artificial intelligence is not limited to rational thinking. Graphical thinking can also promote the development of artificial intelligence. Mathematics is often regarded as the basic science of many subjects. Mathematics is also embedded in the field of language and thinking. The subject of artificial intelligence should also borrow mathematical tools. At present, the types of technologies used in the judicial field are almost the same. Most of them are based on deep learning, natural language processing, text recognition, image recognition, and other technologies. Depending on the scale of data "learned," the depth and accuracy of the generated content are also different. In terms of content types, they are more inclined to digital or logically related information. Table 1 shows a typical case of artificial intelligence applications in foreign countries.

This article strives to introduce artificial intelligence collection, analysis, and application modes on the basis of traditional data statistics to meet the needs of judicial reform in the artificial intelligence era. Traditional data collection is limited in physical space, and information that can be collected is limited. Artificial intelligence technology can transcend the physical space of traditional technology, gain more information, capture track human actions in real time, and can also find information in the physical space. More external data details can be found in [6].

\section{The Current Status of Artificial Intelligence in the Judicial Field and the Method of Use}

\subsection{The Value and Disadvantages of Artificial Intelligence in} the Judicial Field. From ancient times to the present, seeking advantages and avoiding disadvantages are human instincts, so people hope that they can foresee before the arrival of social phenomena in order to make preparations and preventive measures [7]. This problem also extends to the judicial field. Because the space and time of the occurrence of cases are irreversible, humans do not have the ability to predict and prevent the occurrence of cases and can only deal with cases after they have occurred [8]. If the rights and public rights of the suspects and the parties to the case are placed on two opposing angles, generally speaking, postevent processing will treat the rights of the people as victims [9]. Background processing has a backlog, which will infringe on the health and property rights of citizens. The use of artificial intelligence spy technology in case analysis helps to find the law of the activities of various cases in a large number of historical cases. Judicial staff can predict and understand the time, place, crowd, and method of a particular case based on the results of available analysis, then try to identify and find the case before it happens, and finally assign and use judicial resources' prevention and precautionary measures. The application of artificial intelligence technology in the judicial field is conducive to gradually replacing postprocessing methods with preprocessing methods, thereby avoiding the occurrence of cases as much as possible, protecting citizens' property safety and personal freedom, and ensuring national social stability and national security.

With the application of artificial intelligence technology in the judicial field, we usually ask the following question: how much data does the judicial department have? At this stage, the legal construction of personal information protection in the judicial process in our country is still blank [10]. The reasons include the following two points. One is that privacy protection laws are still incomplete. Second, the judicial system lacks management regulations for the protection of personal privacy. Lack of rules in both cases makes it difficult for personal information to be effectively protected during the investigation [11]. While privacy policy does not exist, third-party industry regulations have not yet been enacted. The third group referred to here mainly refers to artificial intelligence companies, while third-party industrial regulations refer to the industrial regulations of non-spyware companies. Retrieving data from artificial intelligence companies is an important part of the establishment of judicial systems. In fact, artificial intelligence companies are of great significance to the establishment of judicial artificial intelligence systems, and they have also driven the national artificial intelligence strategy forward.

The definition of black-box effect (black box) is to input data into a system, and the system obtains the result after data analysis and data processing. The entire analysis and processing process is not open to the outside world and is in a state of complete confidentiality. It is usually used to describe the behavior of the human brain, the transistor, and certain algorithms [12]. Because this mechanism is completely opaque, it is difficult to modify algorithm errors and data errors, so that professional maintenance personnel cannot accurately locate the error location. Therefore, many professional researchers also refer to the analysis of artificial intelligence and decision making as a "black-box effect," meaning that the analysis of hypocrisy and ethical decision making is not visible. External employees can only store input data and output data, but they have no way to know how to calculate and how to work. Because this method is completely opaque, it is difficult to change algorithm errors and data errors, so that professional repair technicians can accurately locate the error location. We can imagine that if China builds an early warning system for high-risk elements with artificial intelligence, it uses artificial intelligence 
TABLE 1: Typical cases of foreign artificial intelligence applications.

\begin{tabular}{|c|c|c|c|}
\hline Company name & Product & Technology & Application \\
\hline Automated Insights & Wordsmith & $\begin{array}{c}\text { Natural language division } \\
\text { Management, deep } \\
\text { learning } \\
\text { Text recognition } \\
\end{array}$ & $\begin{array}{l}\text { The Associated Press used to compile and distribute corporate } \\
\text { financial reports }\end{array}$ \\
\hline Give Me Sport & Give Me Sport & $\begin{array}{l}\text { Deep learning, text } \\
\text { Word recognition }\end{array}$ & Filter spam and write sports news \\
\hline Washington Post & Heliograf & $\begin{array}{l}\text { Deep learning, text } \\
\text { Word recognition }\end{array}$ & Sports news with reported numbers as the core \\
\hline Narrative Science & Quill & $\begin{array}{c}\text { Natural language division } \\
\text { Management, deep } \\
\text { learning } \\
\text { Text recognition } \\
\end{array}$ & Machine automatic narrative \\
\hline $\begin{array}{l}\text { The New York } \\
\text { Times }\end{array}$ & $\begin{array}{l}\text { Vision API } \\
\text { tools }\end{array}$ & Image identification & Recognize image characters and match text information \\
\hline The Guardian & \#Open001 & $\begin{array}{l}\text { Deep learning, text } \\
\text { Word recognition }\end{array}$ & $\begin{array}{c}\text { Collecting and reporting based on information in social networks } \\
\text { Tao }\end{array}$ \\
\hline
\end{tabular}

algorithms to guess a person's crime probability and implement related methods. According to such mechanism, whether a person is suspected of a crime and whether a case needs to be filed is often determined by the artificial intelligence algorithm. If there is an error in the data source or algorithm, if someone is mistakenly regarded as a criminal suspect, their human rights will be violated. The right to freedom will also seriously damage their dignity [13]. Even worse, this artificial intelligence investigation mechanism is not public. Everyone is being censored secretly. They cannot participate in the artificial intelligence decision-making process. They do not know whether they are on the blacklist or whether they know which piece of data will trigger the artificial intelligence algorithm system.

2.2. Comparison of Various Algorithms of Artificial Intelligence. There are many artificial intelligence algorithms used in judicial analysis. Widely used are artificial neural network, simulated annealing method, genetic algorithm, particle swarm algorithm, expert system, and so on. In the reconstruction of distribution network, the application of ANN has certain advantages [14]. On the one hand, the algorithm has a strong ability to map nonlinear relationships. On the other hand, during reconstruction, ANN does not use power flow calculation and loss reduction estimation; SA algorithm was proposed by Metropolis et al. in 1953 and was first effectively applied in combinatorial optimization problems by Kirkpatrick et al. Genetic algorithm was first proposed by Professor J. Holland in 1982. In reconstruction, the state of the switch is represented by coded bits [15]. Particle swarm algorithm was put forward in 1995 and has received a lot of attention. The literature uses binary coding and uses the PSO algorithm to reconstruct the problem [16]. The expert system is based on heuristic rules and has high requirements for the perfection of the knowledge base and the meticulous reasoning mechanism. There are many kinds of artificial intelligence algorithms. This article combines these algorithms [17], learns from each other's strengths, and makes use of their respective advantages to achieve better computing results. Table 2 compares several commonly used algorithms mentioned above in several aspects, such as whether they are globally optimal.

2.3. Artificial Intelligence Neural Network. The algorithm of artificial intelligence neural network to evaluate the current status of the judicial field and its countermeasures is composed of three layers: input layer, hidden layer, and output layer, and the power connection between each layer is realized. Learning consists of four processes: "mode forward propagation," "error backpropagation," "memory training," and "learning convergence." The calculation formula of the output node output $y$ is as follows:

$$
y=i\left(\sum_{x} N o-\theta_{x}\right),
$$

where $y$ is the output of the output node, $N$ is the connection weight, and the node threshold is $\theta_{x}$.

$$
m=f\left(\sum_{x} R X-\theta_{1}\right)
$$

where $m$ is the output of the hidden node, the connection weight is $R$, and the node threshold is $\theta_{1}$. Error of all samples is $F=\sum_{L=1}^{V} e \leq \ell$, and the error of one sample $e=\sum_{i=1}^{m}\left|y_{1}-y_{2}\right|$, where $v$ is the number of samples and $i$ is the output node; then,

$$
\varsigma=\left(i_{1}-y_{1}\right) \times y \times(1-i) .
$$

The weight correction value formula is as follows:

$$
U=R(k)+\eta \pi \lambda
$$

where $k$ is the number of iterations. The threshold correction formula is as follows:

$$
K(\theta+1)=\theta(k)+\eta \lambda
$$


TABLE 2: Algorithm comparison.

\begin{tabular}{|c|c|c|c|c|}
\hline Algorithm & Relies on initial structure & High speed & Global optimal & Power flow calculation \\
\hline Neural networks & $\mathrm{N}$ & $\mathrm{Y}$ & $\mathrm{Y}$ & $\mathrm{N}$ \\
\hline Particle swarm algorithm & $\mathrm{N}$ & $\mathrm{Y}$ & $\mathrm{N}$ & $\mathrm{N}$ \\
\hline Genetic algorithm & $\mathrm{N}$ & $\mathrm{N}$ & $\mathrm{Y}$ & $\mathrm{Y}$ \\
\hline Mathematical optimization techniques & $\mathrm{N}$ & $\mathrm{N}$ & $\mathrm{Y}$ & $\mathrm{Y}$ \\
\hline Simulated annealing & $\mathrm{N}$ & $\mathrm{N}$ & $\mathrm{Y}$ & $\mathrm{Y}$ \\
\hline Optimal flow mode algorithm & $\mathrm{N}$ & $\mathrm{Y}$ & $\mathrm{N}$ & $\mathrm{Y}$ \\
\hline Expert system & $\mathrm{Y}$ & $\mathrm{Y}$ & $\mathrm{N}$ & $\mathrm{N}$ \\
\hline
\end{tabular}

The correction formula from the input node to the hidden node is as follows:

$$
\rho=y(1-y) \sum_{1} \mu_{1} T_{K}
$$

where $y$ is the output of the hidden node, the connection weight is $\mu_{1}$, and the node threshold is $T_{K}$. The weight correction formula from the input node to the hidden node is as follows:

$$
w_{i j}=(k+1) \int w(k)+\eta \lambda \gamma
$$

The number of iterations from the input node to the hidden node is $k$. The formula after threshold correction is as follows:

$$
\theta(s+1)=\theta(k)+\sum_{i+q} \rho \lambda
$$

According to Kirchhoff's law:

$$
E_{i}=V_{i-1}-V_{i},
$$

where $E_{i}=\eta \lambda \gamma, V_{i-1}, V_{i}$, respectively, represent the influencing factors of hidden nodes and the influencing factors of output nodes; according to KCL,

$$
p=\left\{\frac{j+j_{l(n+2)}}{-i}\right\}+Z I .
$$

Then, according to the steps of the calculation of energy flow, first calculate $j$ with $S w=V I n$; second, calculate $E$ with formula (9); then, calculate $p$ by Kirchhoff's law; and finally judge whether it had changed. If not satisfied, go to the next iteration.

$$
P=\sum_{x=i+1}^{i} e j+\sum_{x=i+1}^{i} p k .
$$

\subsection{Basic Particle Swarm and Binary Particle Swarm} Algorithm. The basic particle swarm algorithm is to simulate the foraging behavior of a flock of birds. Every bird changes its speed and location at any time to search for food, and before reaching the goal, the following three rules need to be followed: (1) try to avoid collisions with other birds; (2) maintain a coordinated speed with nearby birds; and (3) try to approach the target group. [18]. The group extremum is the global optimal solution. The basic concept of a basic particle swarm algorithm states that in each iteration, each particle continuously renews its speed and position in the solution area according to the formula and continuously approaches the area indicated by the two extreme values.

$$
Q_{i+1}=\sum_{n} P m+\sum_{j=n+1}^{n} u+V .
$$

The chaotic variable is ergodic, that is, it can search every state in the space without repeating it. The randomness of confounding variables means that the turbidity variables are as messy as random variables. The chaotic variable is very sensitive to the initial conditions, and a very small change in the initial value will also cause a huge change in the later motion, which is very far from it. Because of these characteristics, chaos-based search technology is more advantageous [19]. $L$ mapping is taken as an example to illustrate the basic properties of the mixing motion. The well-known $L$ mapping equations are shown in equations (13) and (14):

$$
\begin{aligned}
& L J_{1}=\frac{R\left(M+N^{2}\right)}{\left|V_{i+1}\right|}, \\
& L q=\frac{m\left(O+N^{2}\right)}{\left|V_{n+1}\right|} .
\end{aligned}
$$

When the binary particle swarm algorithm is applied to the optimization function, the convergence speed is fast, simple, and convenient. The algorithm only has the parameter $w$ to affect the convergence speed of the particle swarm [20]. However, because the particles always fly towards the best position of themselves and the group, the information flows in one direction, and it is easy to fall into the local optimum [21]. It also reduces the success rate of obtaining the optimal solution. Therefore, experts and scholars have developed algorithms to improve the performance of various particles, which not only combine quickly to be a good solution but also reduce the chance of falling into the right place. In recent years, there have been many improvements to the particle swarm algorithm, one of which has improved the weight of inertia. The inertia weight $w$ is the embodiment of the particle's ability to inherit the previous speed and is the representative of the particle's flying tendency. The selection of the inertia weight has an important influence on the algorithm. The particle swarm algorithm has many improvement directions, one of which is the improvement of mixing other technologies, and the 
reason is the omission of the speed parameter in the formula [22]. In this paper, a set of confounding variables is introduced into the inertia weight of the particle swarm algorithm, and the inertia weight formula after the introduction is shown in the following equation:

$$
j=\left[P R+Q M-\frac{1}{2} V-\left(R^{2}-X^{2}\right)\right]^{2},
$$

where $P R$ represents the load power of node $j$ and $M$ and $O$ represent branch losses. Finally, the backpropagation algorithm is used to calculate artificial intelligence. All known data collected and processed by artificial intelligence can be input into the application as a network parameter. After a lot of training, each layer of the network is realized. [23]. The main influencing factors affecting justice are processed accordingly and then input into the program as parameters of the input layer. The link authority realized by artificial intelligence can simulate the operation of artificial intelligence in the judicial field.

\section{Experimental Design of Artificial Intelligence in the Judicial Field}

3.1. Subjects. The subjects of this experiment are mainly selected from various departments in the judicial field. The data are mainly about the differences in the processing of various events in the intelligent construction of various departments compared with the previous traditional processing, as well as the investigation and statistics of the convenience and possible harm brought by artificial intelligence in the process of intelligentization by the personnel of various departments.

\subsection{BP Neural Network Assessment Process of Judicial} Influence. Based on artificial intelligence, the power flow calculation is carried out, and a hybrid optimization algorithm combining turbidity technology and binary particle swarm optimization algorithm is proposed to be used in the algorithm calculation [24]. In the article, small losses of the implant technology network are optimized for objective work, and the feasibility and accuracy of the test algorithm is tested with two examples. Among them, the algorithm of artificial intelligence neural network to evaluate the operation status and countermeasures of the judicial field is composed of three layers: input layer, hidden layer, and output layer, and the power connection between each layer is realized. Its learning consists of four processes: "mode propagation," "error backpropagation," "memory training," and "learning convergence" [25]. To establish a judicial evaluation model, the main factors that affect judicial evaluation can be selected as the input layer parameters, and the output layer parameters are the factors of the evaluation. The process of using BP neural network to assess judicial impact is shown in Figure 1:

3.3. Statistics. All data analysis in this article uses SPSS19.0, statistical test uses two-sided test, significance is defined as
0.05 , and $p<0.05$ is considered significant. The statistical results are displayed as mean \pm standard deviation $(x \pm \mathrm{SD})$. When the test data obey the normal distribution, the double $T$ test is used for comparison within the group, and the independent sample $T$ test is used for comparison between the groups. If the regular distribution is not sufficient, two independent samples and two related samples will be used for inspection.

\section{Experimental Analysis}

4.1. The Influence Mode and Mechanism of Artificial Intelligence on the Judicial Field. As early as in ancient times, there are stories about making robots to help people work in Greek mythology. Since its birth, the concept of artificial intelligence has embraced the good wishes of mankind, which is completely different from what mankind has gained through work for thousands of years. The concept of "getting something for nothing" may become possible [18]. After 2010, artificial intelligence has entered a real explosion period. The maturity of big data and algorithms has greatly promoted the development of artificial intelligence.

It can be seen from Figure 2 that if the Internet environment provides fertile soil for artificial intelligence, then artificial intelligence is the seed, and deep learning algorithms are the fertilizers. The three are indispensable. At the same time, the Internet, artificial intelligence, and in-depth learning all point to the same new concept, or in other words, thought communication. At the same time, ethics and morality are two concepts in philosophy, and ethics and morality are essentially norms. Industrial ethics often refers to the moral ethics and conscience embodied in the process of industrial development.. With the widespread use of artificial insemination technology in the judiciary, a number of regulatory issues will arise. Philosophical ethics and legal issues cannot be effectively solved by relying solely on philosophers and legal experts. They require individual participation of industry workers. The establishment of the new rules is the foundation of whether artificial intelligence technology can be applied to specific industries or not, and it is also the threshold that must be crossed to realize the intelligent transformation and upgrading of the judicial field. Discussions about artificial intelligence at the philosophical level are also very intense, and issues related to ethics and morality are even more controversial.

As shown in Table 3, we can divide the level of automation in moral decision making into four levels. Does the intelligent machine have "awareness" of its creation, can it be the criterion for determining whether it owns the work? As human beings, how should we view the ownership of artificial intelligence creations, whether we own them or take charge of them? Artificial intelligence is becoming more and more "intelligent." Robots are full of emotion and consciousness. It seems that it is not so far away. At the same time, it also brings uncertainty to social development, which leads to complex ethical and safety issues. While the development of the judicial field focuses on technological progress, it is also necessary to pay attention to the relationship between artificial intelligence and new rules, 


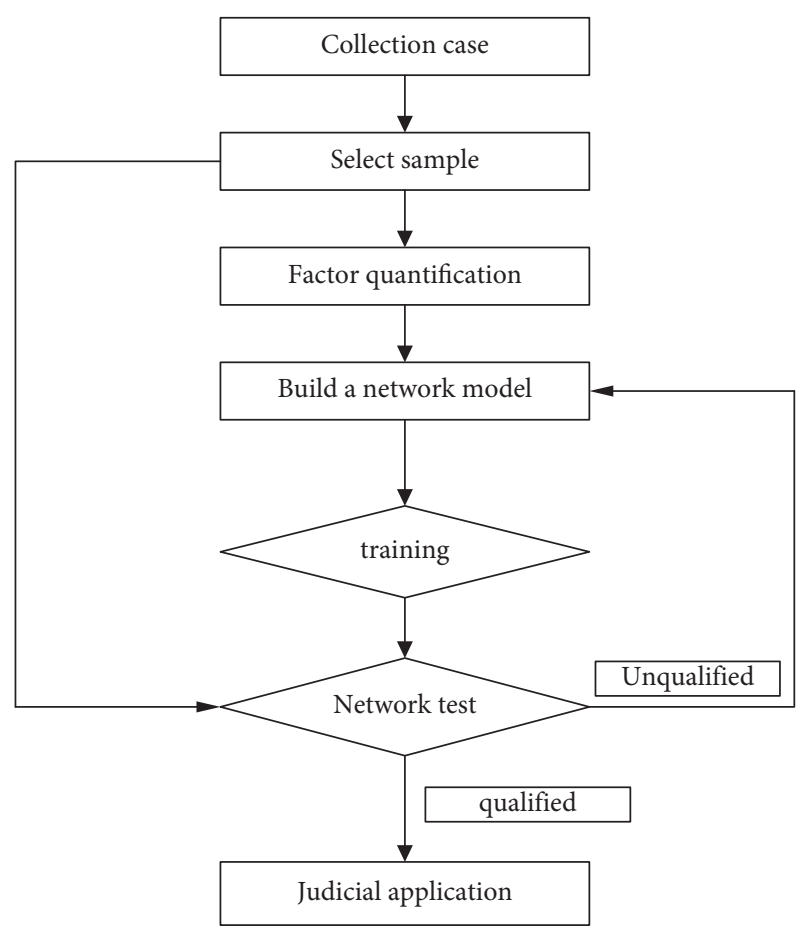

FIgURE 1: Artificial intelligence neural network evaluation program.

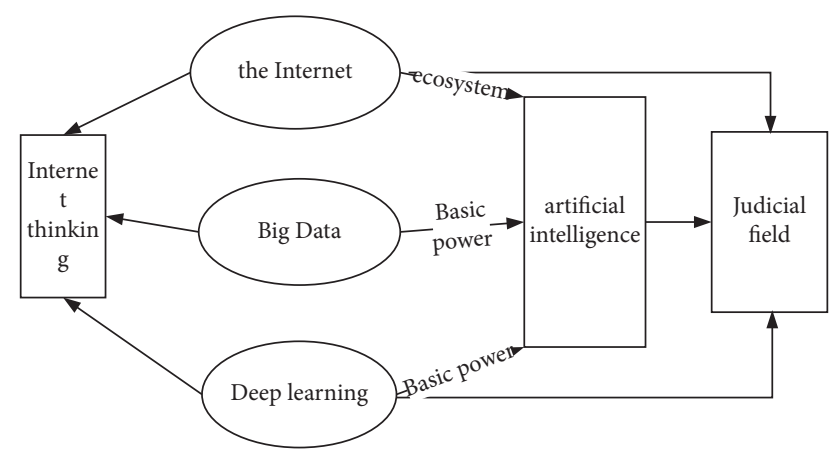

FIgUre 2: The impact of artificial intelligence on the judicial field from the conceptual level.

especially at the stage when the whole society has not yet formed a unified standard cognition. Only by introducing technology and innovative changes within the rules can we achieve long-term and effective development in the future.

4.2. Artificial Intelligence Improves Judicial Efficiency. The court needs an appropriate time period for the conclusion of each case. If the trial time of the case is too short, there may be omissions in a certain link. If the trial time of the case is too long, it is difficult to guarantee the rights and interests of the parties, and it will also consume too much resources of the parties and society. Therefore, timely warnings on all aspects of legal approval using artificial intelligence can effectively avoid random or outdated trials and allocate social grants accordingly.
It can be seen from Figure 3 that the time for case trials in our country is still too long. In 2017, there were more than 8 million first-instance civil cases, but only 100,000 cases were closed. However, in 2019, with the construction of smart courts, the situation has been greatly improved. In 2020, there will be 12.65 million first-instance cases and 1.2 million closed cases. It can be seen that with the popularization of artificial intelligence, judicial efficiency has been greatly improved.

From Figure 4, we can see that with the construction of smart courts, artificial intelligence analyzes big data and decides not to prosecute 140,000 cases, and over 36,500 cases of illegal and evidence collection have been supervised and corrected. It can be seen that the popularization of artificial intelligence can effectively prevent crimes. Behaviors can also better correct wrongful cases and irregular behaviors in the judicial field, which is conducive to judicial justice.

4.3. Suppressing Duty Crimes in the Judicial Field. Current judicial investigations show that with the continuous increase in anti-corruption and crime fighting, this has a great deterrent effect on public servants in the judicial field to commit crimes on duty. However, due to the complex and hidden characteristics of judicial crimes and the social nature of China's human relations, the duty crime rate in the judicial field has always been at a high level.

It can be seen from Table 4 that duty crimes in the judicial field have been increasing in a certain city in recent years. Among them, the number of duty crimes committed by judges and police officers is much higher than that of prosecutors. Ordinary people deal with complex characters and are more tempted than prosecutors. However, the procuratorial system generally only has institutions at the county level and above, with concentrated personnel and fewer contacts.

It can be seen in Table 5 that there were 16 people who announced the test, and the number of people applying for the test was $52 \%$ of the total number of people involved. Among them, there are 15 people in cases where the crime rate is less than 100,000 yuan, 5 people who have been sentenced to life imprisonment, and 10 people who are being investigated. There are 16 people in cases where the crime rate is more than 100,000 yuan, 10 people who have been sentenced to life imprisonment, and 6 people who are being investigated. In judicial judgments, due to the interpersonal relationship and strong social pressure, the judgments of duty crimes in the judicial field are usually weak, and it is difficult to play a deterrent effect. On the contrary, it exacerbates the situation of duty crimes in the judicial field. The application of artificial intelligence to judgments in the judicial field, because the machine is objective and stable, will not be interfered by external factors and the temptation of money. It can not only avoid the influence of subjective judgments on the judgment of the case but also prevent judicial personnel from accepting bribes and going astray. 
TABle 3: Automation level description.

\begin{tabular}{lc}
\hline Automation level & Description \\
\hline $\begin{array}{l}\text { Manual control } \\
\text { Consensual control }\end{array}$ & Human must do everything without assistance from automation \\
$\begin{array}{l}\text { Monitored control } \\
\text { Full automation }\end{array}$ & Automation offers suggestion and acts only with user consent \\
\hline
\end{tabular}

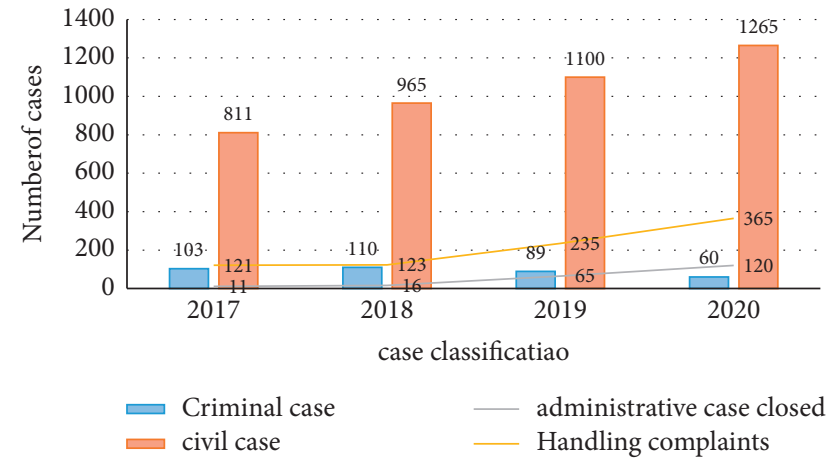

Figure 3: Diagram of cases closed in courts at all levels.

4.4. Drawbacks of Artificial Intelligence to the Judicial Field. In the era of artificial intelligence, the traditional privacy protection model can no longer protect private information. "Information and permission" is the main method of traditional privacy protection. The relevant rights protection mechanism mainly protects the data collector. The related obligations and responsibilities are on the data collector, but there is a lack of clear provisions on the use of data. Many scholars believe that in the era of artificial intelligence, it is necessary to construct a new privacy protection method that is completely different from the previous privacy protection model, especially to restrict behaviors such as in-depth mining and analysis. However, if implemented in this way, it will inevitably lead to a significant decrease in the value of artificial intelligence. In the era of artificial intelligence, we must seek a balance between the use and protection of personal information. The realization of the value of artificial intelligence requires the protection of personal privacy as the basic condition to establish industry standards for data retrieval. The so-called data recovery methods refer to the control of the behavior of third parties in the justice system when they perform data retrieval. Of course, industry regulations formulated by relevant intelligence agencies can play a comprehensive role in monitoring industry data, which is also a requirement for third parties to retrieve information in accordance with relevant laws and regulations.. Based on the hierarchical and classified management of data, artificial intelligence companies can connect the hierarchical management of data with the procedures of judicial organs to retrieve data and can formulate different retrieval requirements according to the difference of information privacy, as shown in Figure 5.

First, we must first clarify that personal information can only be obtained when the state department is fighting crime or protecting national security, and the data subject's right to know must be fully protected. The court must tell if the relevant data are retrieved as evidence. In related criminal proceedings, if it is necessary to obtain evidence from the parties, based on the data subject's control of personal information, the artificial intelligence company should allow it; if the relevant data of others is involved, an application can be submitted to the review authority, and the people's court approves the acquisition [24]. In civil or administrative litigation, when a party or his agent needs to retrieve, only his own data can be approved; relevant specific data related to other persons can be obtained by applying to the court. Second, for specific objects of public data and semipublic data, the national judicial organs can have the authority to retrieve the corresponding personal data, but the parties must be informed of these circumstances. And in the relevant litigation, if you want to obtain the relevant data of the parties, the artificial intelligence company can approve the acquisition. If the relevant data of others are involved, the defense lawyer can provide relevant application instructions and obtain the consent of the parties involved before the retrieval; it is the same for civil litigation and administrative litigation parties to obtain evidence. Third, for public data, it can be retrieved through public channels.

4.5. Coordinating the Balanced Relationship between Intelligent Judgment and Human Brain Refereeing. The essence of judicial judgment is to analyze and judge cases, obtain useful clues from complex evidence and information, and deal with specific cases in an abstract system. Judicial judgment is a process by which judges make subjective judgments on the background of the case and the suitability of relevant laws. Each person's social experience and emotional differences may bias the results and have strong subjective initiative. Artificial intelligence is an abstract experience derived from the calculation of data through algorithms. The judgment is fixed and is not affected by emotions and other factors. It only follows the objective calculation results. Although the judgment results are objective, they are due to different cases. The background details are different, and the same result may not be suitable for the same type of case to a large extent. After all, justice can take on different shapes and have very different looks at any time [25]. In different situations, justice may be completely opposite. This requires judges to make comprehensive considerations in the complex social background and different individual circumstances to make judgments, but machine judgments are indiscriminate judgments that do not consider external factors. Subjective and objective are both necessary and necessary in the trial of a case, and this highlights the contradiction between the machine brain and the human brain in the judgment. How 


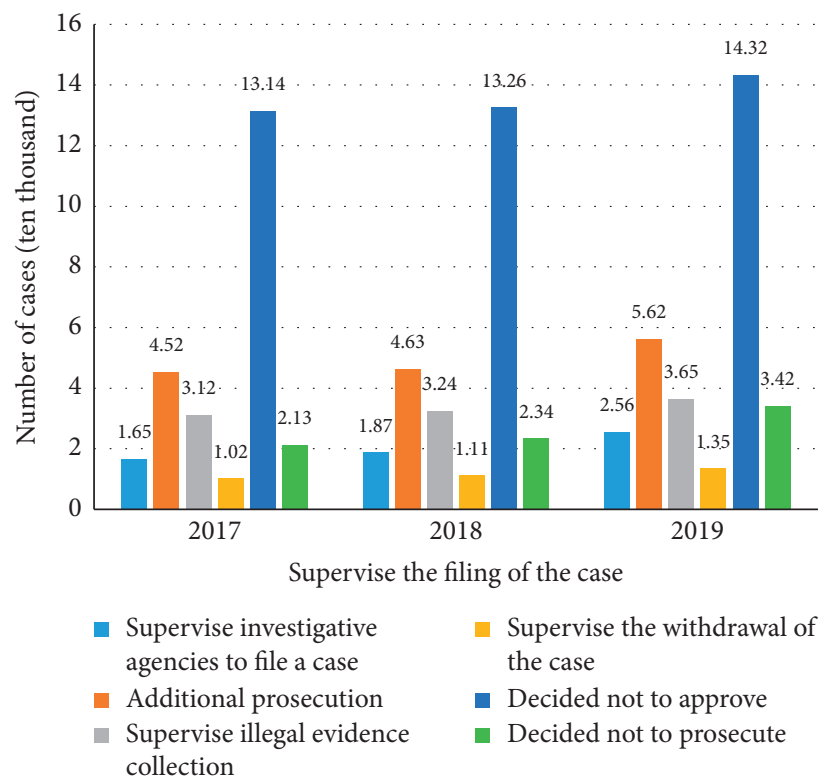

FIGURE 4: Statistics chart of investigation and filing of the national inspection and erasure system.

TABLE 4: Situations related to duty-related crimes by judicial officers in a certain city.

\begin{tabular}{lcccc}
\hline Year & & \multicolumn{2}{c}{ Organ } & \\
& Public security (person) & Court agency (person) & Inspection agency (person) & Total (people) \\
\hline 2017 & 11 & 2 & 0 & 14 \\
2018 & 17 & 3 & 2 & 20 \\
2019 & 14 & 5 & 0 & 21 \\
2020 & 15 & 2 & 3 & 72 \\
Total & 57 & 12 & & 72 \\
\hline
\end{tabular}

TABLE 5: Probation and probation of judicial officers for duty crimes in a certain city.

\begin{tabular}{lcccc}
\hline Amount of crime & Number of people involved & Sentenced & Number of probation & Probation rate $(\%)$ \\
\hline $50,000-100,000$ yuan & 15 & 5 & 10 & 60 \\
More than 100,000 yuan & 16 & 10 & 6 & 37 \\
Total & 31 & 15 & 16 & 52 \\
\hline
\end{tabular}

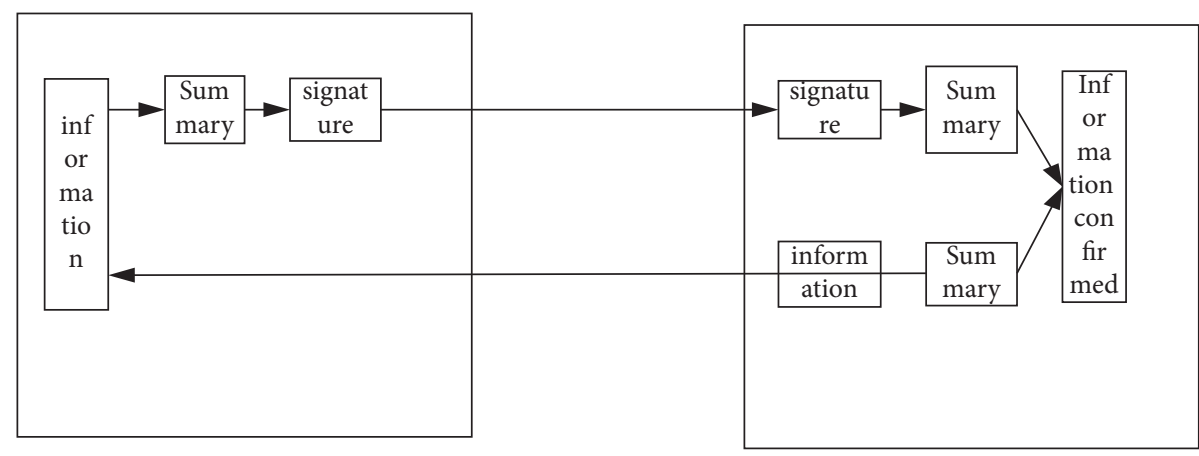

FIGURE 5: User information retrieval program.

to eliminate this contradiction is a point that needs to be considered in the follow-up development.

According to Figure 6, the machine brain is only a mechanical integration of massive case experience, and it can only converge to the same infinitely, specifically to cases with different backgrounds. Even cases of the same background may differ due to the differences between different parties and judges, especially when it comes to 


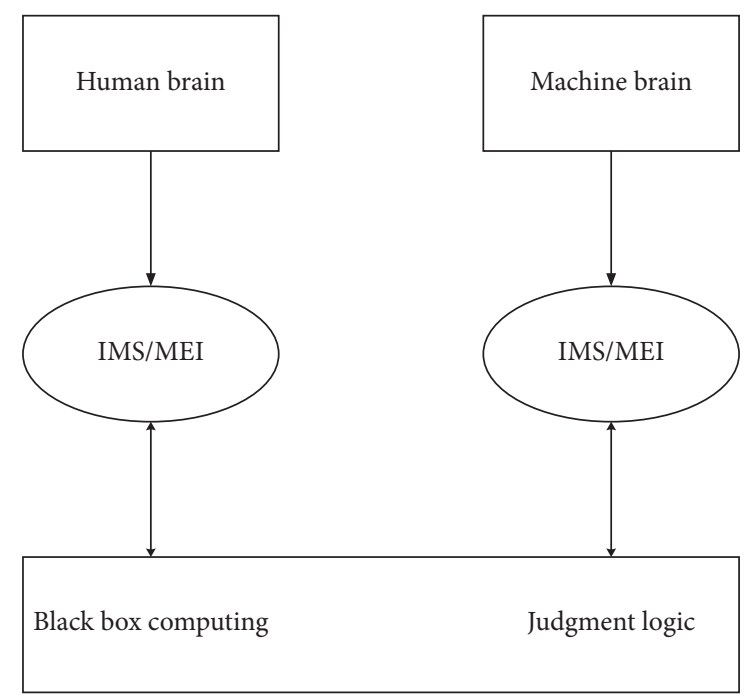

Figure 6: Judgment mechanism of human brain-computer.

some philosophical aspects, such as different perceptions of freedom and the right to life. Different judgments are made, so these characteristics make machine brain technology more suitable for auxiliary work of judicial judgments.

\section{Conclusion}

Studies have shown that artificial intelligence has a great effect on the judicial field, which can greatly improve the efficiency of courts and ensure the rights and interests of the parties. At the same time, due to the objectivity of artificial intelligence, it can avoid judicial crimes caused by human handling in dealing with related issues and improve the fairness of justice and the construction of clean government in our country. However, artificial intelligence may also bring about the risk of personal information leakage. As its algorithmic calculations have black-box effects, they may also bring about differences in fairness. This requires us to establish relevant laws and regulations as soon as possible to protect citizens through laws. At the same time, due to the mechanical and objectivity of artificial intelligence judgments, it is impossible to effectively make differentiated judgments based on the background of different cases, so it may be more suitable for the auxiliary role of the human brain.

\section{Data Availability}

No data were used to support this study.

\section{Conflicts of Interest}

The authors declare that they have no conflicts of interest.

\section{References}

[1] P. Havinga, N. Meratnia, and M. Bahrepour, Artificial intelligence based event detection in wireless sensor networks, vol.
85, no. 6, , pp. 1553-1562, University of Twente, Enschede, Netherlands, 2017, PhD Thesis.

[2] G. Pigozzi, A. Tsoukias, and P. Viappiani, "Preferences in artificial intelligence," Annals of Mathematics and Artificial Intelligence, vol. 20, no. 3-4, pp. 1-41, 2016.

[3] L. Rongpeng, Z. Zhifeng, Z. Xuan et al., "Intelligent 5G: when cellular networks meet artificial intelligence," IEEE Wireless Communications, vol. 24, no. 5, pp. 175-183, 2017.

[4] F. Goyache, J. J. Del Coz, J. R. Quevedo et al., "Using artificial intelligence to design and implement a morphological assessment system in beef cattle," Animal Science, vol. 73, no. 01, pp. 49-60, 2016.

[5] C. Krittanawong, H. Zhang, Z. Wang, M. Aydar, and T. Kitai, "Artificial Intelligence in precision cardiovascular medicine," Journal of the American College of Cardiology, vol. 69, no. 21, pp. 2657-2664, 2017.

[6] G. Luis, M. Glen, P. Eduard et al., "Unmanned aerial vehicles (UAVs) and artificial intelligence revolutionizing wildlife monitoring and conservation," Sensors, vol. 16, no. 1, p. 97, 2016.

[7] L. D. Raedt, K. Kersting, S. Natarajan, and D. Poole, "Statistical relational artificial intelligence: logic, probability, and computation," Synthesis Lectures on Artificial Intelligence and Machine Learning, vol. 10, no. 2, pp. 1-189, 2016.

[8] S. Jha and E. J. Topol, "Adapting to artificial intelligence: radiologists and pathologists as information specialists," Jama, vol. 316, no. 22, pp. 2353-2354, 2016.

[9] L. M. Prevedello, B. S. Erdal, J. L. Ryu et al., "Automated critical test findings identification and online notification system using artificial intelligence in imaging," Radiology, vol. 285, no. 3, pp. 923-931, 2017.

[10] M. Moravík, M. Schmid, N. Burch et al., "Deepstack: expertlevel artificial intelligence in no-limit poker," Science, vol. 356, no. 6337, p. 508, 2017.

[11] H. Lu, Y. Li, C. Min et al., "Brain intelligence: go beyond artificial intelligence," Mobile Networks and Applications, vol. 23, no. 7553, pp. 368-375, 2017.

[12] D. Hassabis, D. Kumaran, C. Summerfield, and M. Botvinick, "Neuroscience-inspired artificial intelligence," Neuron, vol. 95, no. 2, pp. 245-258, 2017.

[13] A. Jeavons, "What is artificial intelligence?" Research World, vol. 2017, no. 65, 75 pages, 2017.

[14] E. Sulistyawati and E. F. Tihurua, "Analysis of the condition of forest interior and edge in Mount Papandayan, West Java, Indonesia based on floristic composition and structural characteristics of tree community," Biodiversitas Journal of Biological Diversity, vol. 20, no. 3, pp. 900-906, 2019.

[15] О. В. Сирота, "Comparative analysis of the methods of expression of negation in the Russian and Romanian languages (based on literary texts," Мова, nо. 29, pp. 110-114, 2018.

[16] S. Zhou, B. Chen, Y. Zhang, H. Liu, Y. Xiao, and X. Pan, "A feature extraction method based on feature fusion and its application in the text-driven failure diagnosis field," International Journal of Interactive Multimedia and Artificial Intelligence, vol. 6, no. 4, p. 121, 2020.

[17] S. Dygnatowski, P. Dygnatowski, and Ł. Domżał-Drzewicki, "The analysis of using structural solutions in cybersecurity based on orchard operation," Journal of Konbin, vol. 49, no. 1, pp. 281-298, 2019.

[18] B. Zohuri and E. Ooi, "Halting the pandemic -vaccine and vaccination a realistic path forward," European Journal of Pharmaceutical Sciences, vol. 5, no. 4, pp. 91-99, 2021. 
[19] S. Blusseau, A. Carboni, A. Maiche, J. M. Morel, and R. Grompone Von Gioi, "Measuring the visual salience of alignments by their non-accidentalness - sciencedirect," Vision Research, vol. 126, pp. 192-206, 2016.

[20] L. Xie, D. Ding, Z. Wei et al., "Moving time UCAV maneuver decision based on the dynamic relational weight algorithm and trajectory prediction," Mathematical Problems in Engineering, vol. 2021, no. 4, 19 pages, Article ID 6641567, 2021.

[21] X. Shi and J. Zhang, "Analysis of Application of tennis electronic referee based on artificial intelligence in tennis matches," in Proceedings of the 2020 International Conference on Artificial Intelligence, Computer Networks and Communications (AICNC 2020) 27-30, Lijiang, China, December 2020.

[22] H. Lee, "An analysis of research trend in women's retirement: based on the publications in academic journals and theses," Issues in Feminism, vol. 16, no. 2, pp. 177-214, 2016.

[23] T. A. S. K. Ishwarya, R. C. Appala Naidu, K. Meghana, and G. Prabhakar Reddy, "A modern approach to design and integrate conceptual methods in video games with artificial intelligence," Materials Today: Proceedings, vol. 4, no. 8, pp. 9100-9106, 2017.

[24] W. Shan, S. Zhang, and Y. He, "Machine learning based sidechannel-attack countermeasure with hamming-distance redistribution and its application on advanced encryption standard," Electronics Letters, vol. 53, no. 14, pp. 926-928, 2017.

[25] H. Nini and D. Kong, "Research on the application of children's reading analysis based on artificial intelligence-_take small "raccoon reading" and "jiao jiao reading" as examples," in Proceedings of the 2021 4th International Conference on Advanced Algorithms and Control Engineering (ICAACE 2021), Sanya, China, January 2021. 